\title{
A solid-analytical-based model for extracting cutter- workpiece engagement in 5-axis flank machining
}

\author{
Dongdong $\mathrm{Li}^{1, \mathrm{a}}$, Weimin Zhang ${ }^{1,2}$, Tengfei Shang ${ }^{1}$, Tianhong Gao ${ }^{2}$, Shuo $\mathrm{Liu}^{2}$ and Jürgen Fleischer ${ }^{2,3}$ \\ ${ }^{1}$ School of Mechanical Engineering, Tongji University, 201804 Shanghai, China. \\ ${ }^{2}$ Sino-German Advanced Manufacturing Technology Center (CDHK), Tongji University, 201804 Shanghai, China. \\ ${ }^{3}$ WBK Institute of Production Science, Karlsruhe Institute of Technology, 76131 Karlsruhe, Germany.
}

\begin{abstract}
. 5-axis flank milling is applied extensively in aerospace, die-molds, and automotive industries because of high efficient material removal rate. Commercial CAM software can only simulate the tool path and collision at present, but cannot handle with cutting force during cutting process due to the variable geometrical cutter workpiece engagement (CWE) region of 5-axis milling. This paper presents a novel solid analytical model for extracting the CWE maps. The CWE is obtained analytically by performing Boolean operations between the cutter and inprocess-workpiece (IPW) at any given cutter location (CL) point, instead of using the cutter and the removal volume. The proposed process simulation method could identify the CWE efficiently and precisely for general cutting tools. Finally, the CWE boundaries are mapped from a 3D space into a 2D plane defined by the immersion angle and the axial depth of a given cutter. The proposed solution can be easily integrated into the CAM software for predicting milling force and optimizing parameters in 5 -axis milling.
\end{abstract}

\section{Introduction}

New processes in today's technology, e.g. electrochemical machining and 3D printing have been applied to manufacturing. However, parts machined with 5-axis milling still covers the main proportions in automotive, aircraft parts and molds. 5-axis milling is quite often used for surface machining of impellers, but it still has some defects that need to be optimized. The tool paths and CL file can be generated by commercial CAM. These solutions are only geometrical motion processes, namely, the kinematics properties of the machine tool and the cutting force between cutter and workpiece are not considered for the metal cutting process [1], so products are prone to quality problems. This article provides an optimization solution for processing, and the flow diagram is shown in Figure 1. Accurate modeling and extraction of the CWE area is an extremely important knot of the cutting force prediction model throughout the process.

Various approaches of CNC milling process have been described in previous research works. Kim et al. [2] presented the Z-map method to determine the cutter contact area, and cutting force acting on the engaged cutting edge elements are calculated using an empirical method. Lazoglu et al. [3] did a great study about ball end mill, meanwhile a boundary representation (B-rep) method was developed to find the cutter-workpiece engagement (CWE), which then is used to estimate milling force and

\footnotetext{
${ }^{\mathrm{a}}$ Corresponding author : 1510289@tongji.edu.cn
} 
feedrate optimization. Erdim et al. [4] proposed a new approach to shape representation called composite adaptively sampled distance fields. This function yields the minimum Euclidean distance from a point to the closest in the boundary of the set. Actually, these could be categorized into three major approaches: discrete representations (based on z-buffer, dexels or voxels), solid modeling (Brep, CSG), and point based methods [5].

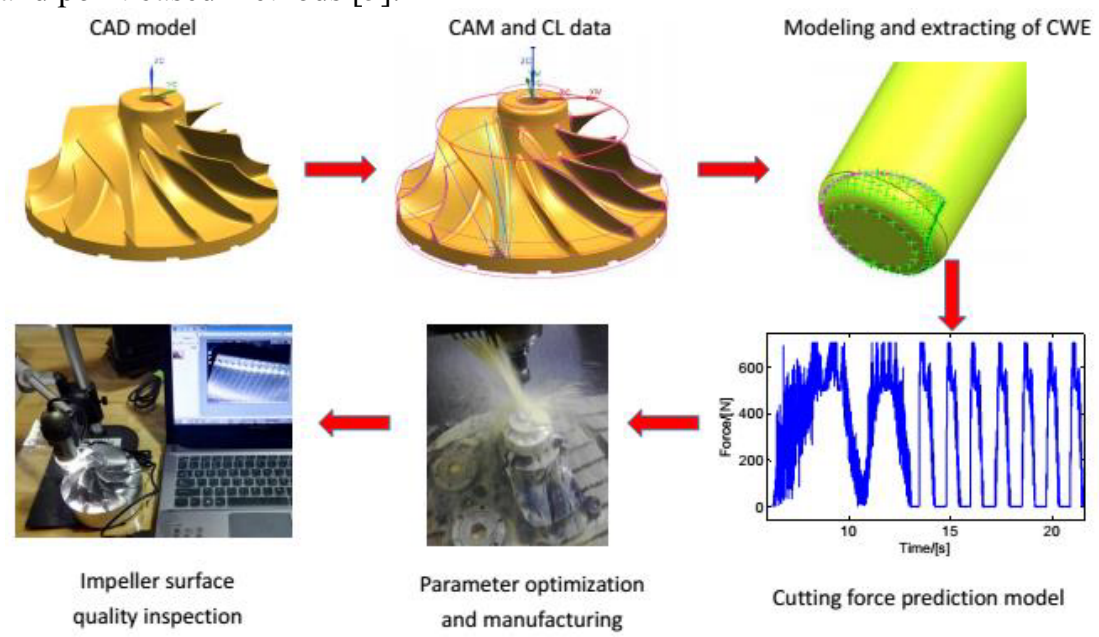

Figure 1. Optimization of 5-axis milling process

According to the Ref. [5,6], the solid model method of CWE extraction has the most accurate results among these methods, though it still has the challenge of computational complexity and cost. This technical trend must be mentioned, that is, the solid modeling solution is greatly promising in industry 4.0 and can be easily integrated into the CAD/CAM softwares.

\section{CWE model for flank milling}

In order to develop CAM, Chiou et al. [7] presented a solution for the determination of instantaneous cutter swept envelope, which provides a feasible tool envelope concept for solid modeling methods. Multiple researchers perform modeling, simulation and optimization of 5-axis NC machining by a ball end mill or a taper ball end mill [2-6,8-10]. However, there are very few CWE extraction studies about 5-axis flank milling in recent years. So a solid analytical model for CWE map in 5-axis flank milling is proposed in this paper. Generally speaking, a bull nose cutter is usually selected for flank milling considering the characteristics of surfaces.

\subsection{Workpiece coordinate system and tool coordinate system}

To accurately describe the cutter movement in 5-axis milling, it is needed to build the workpiece coordinate system (WCS) and tool coordinate system (TCS). On one hand, the CAD model, toolpath generation, and CL file are generally described in the WCS. On the other hand, the CWE and engagement angle are firstly preferred to be illustrated in the TCS. Therefore, the coordinate conversion between the fixed WCS and the instantaneous TCS must be clearly defined before milling process. The toolpath and CL file generated by CAM are shown in Figure 2, the standard format is usually used to describe the cutter trajectories in 5-axis milling, for example, GOTO/x, y, z, i, j, k in each line of CL file that represents the tool tip position and tool axis orientation, respectively. 


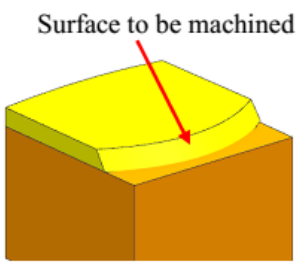

CAD model

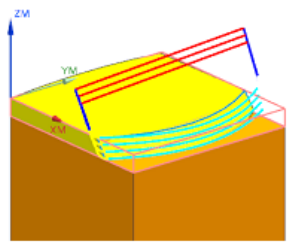

Toolpath in CAM
PAINT/COLOR, 6

FEDRAT/MMPM, 1680.0000

GOTO/-23. 0305, $-55.1666,-42.3660$

GOTO/-22. $9146,-54.6792,-42.2192$

PAINT/COLOR, 31

GOTO/-22. 8783, -54. 2242, $-42.1250,-0.3729354,-0.2505046,0.8934017$ GOTO/-22. 7944, -53.0380, -41.8778, -0. 3895400, -0. 2185397, 0.8947061 GOTO/-22. 7146, -51. 7907, $-41.6144,-0.4017683,-0.2176220,0.8895071$ ............

Figure 2. Generation of the toolpath and CL data in CAM

Schematic diagram of both coordinate systems are shown in Figure 3. The cutter contact (CC) points $\left(x_{c}, y_{c}, z_{c}\right)$ can be calculated from the CL points and cutter geometry [11]. Then the projection point $\left(x_{p}, y_{p}, z_{p}\right)$ of CC points can be calculated by the following equation:

$$
\left\{\begin{array}{l}
x_{p}=x_{c}-r \cdot i \\
y_{p}=y_{c}-r \cdot j \\
z_{p}=z_{c}-r \cdot k
\end{array}\right.
$$

Where $r$ is the radius of cutter end part.

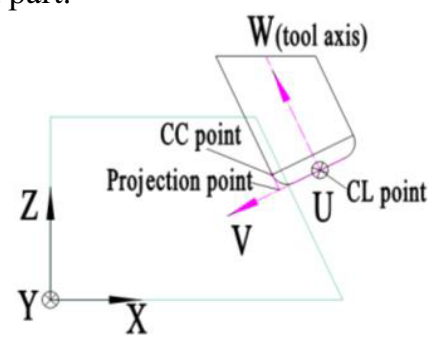

Figure 3. Definition of the TCS

The projection point and the CL point $\left(x_{t}, y_{t}, z_{t}\right)$ are together connected to obtain the $\mathrm{V}$-axis unit vector:

$$
V=\frac{\left[\left(x_{t}-x_{p}\right),\left(y_{t}-y_{p}\right),\left(z_{t}-z_{p}\right)\right]}{\sqrt{\left(x_{t}-x_{p}\right)^{2}+\left(y_{t}-y_{p}\right)^{2}+\left(z_{t}-z_{p}\right)^{2}}}
$$

As a vector cross product of the $\mathrm{V}$-axis and $\mathrm{W}$-axis unit vector, the $\mathrm{U}$-axis unit vector can be expressed as:

$$
U=V \times W=\left[\begin{array}{ccc}
i & j & k \\
V_{i} & V_{j} & V_{k} \\
W_{i} & W_{j} & W_{k}
\end{array}\right]
$$

In the 5-axis flank milling, the coordinate conversion between WCS and TCS contains two parts: the translation transformation and rotation transformation. Homogeneous matrix transformation of 5axis coordinates has already been studied extensively. Thus, the matrix equation can be represented as follows:

$$
T=\left[\begin{array}{cccc}
U_{i} & V_{i} & W_{i} & x_{t} \\
U_{j} & V_{j} & W_{j} & y_{t} \\
U_{k} & V_{k} & W_{k} & z_{t} \\
0 & 0 & 0 & 1
\end{array}\right]
$$

Obviously, the mathematical equation between the WCS and TCS can be written as:

$$
[x, y, z, 1]^{T}=T \cdot[u, v, w, 1]^{T}
$$




\subsection{Surface normal vector of the cutter}

The surface normal vector of a cutter plays a vital role in calculating the feasible CWE area. According to the geometry of a bull-nose cutter, the whole cutter surface can be divided into a cylindrical section and an arc section. An arbitrary point $M\left(u_{1}, v_{1}, w_{1}\right)$ and its surface normal $N\left(n_{i}, n_{j}, n_{k}\right)$ of the cutter in the TCS can be expressed as follows:

For the cylindrical section $\left(w_{1}>r\right)$,

$$
\left\{\begin{array}{l}
M\left(\phi, w_{1}\right)=\left(\frac{D}{2} \sin \phi, \frac{D}{2} \cos \phi, w_{1}\right) \\
N\left(\phi, w_{1}\right)=\left(\frac{D}{2} \sin \phi, \frac{D}{2} \cos \phi, 0\right)
\end{array}\right.
$$

For the arc section $\left(0 \leq w_{1} \leq r\right)$,

$$
\left\{\begin{array}{l}
M\left(\phi, w_{1}\right)=\left(\left(\frac{D}{2}-r+\sqrt{r^{2}-\left(r-w_{1}\right)^{2}}\right) \sin \phi,\left(\frac{D}{2}-r+\sqrt{r^{2}-\left(r-w_{1}\right)^{2}}\right) \cos \phi, w_{1}\right) \\
N\left(\phi, w_{1}\right)=\left(\sqrt{r^{2}-\left(r-w_{1}\right)^{2}} \sin \phi, \sqrt{r^{2}-\left(r-w_{1}\right)^{2}} \cos \phi, w_{1}-r\right)
\end{array}\right.
$$

Where $\phi$ denotes the immersion (engagement) angle of cutting edge at the CL point, and $\phi \in[0,2 \pi]$.

\subsection{Feasible contact surfaces (FCS) on a bull-nose cutter}

Different from the 3-axis milling process, the feed vector $F_{a, b}$ of the cutter is no longer constant in 5axis flank milling due to the additional two rotary motion, here the first subscript $a$ represents the ath cut, and the second subscript $b$ represents the b-th cutting location in the a-th cut. Now, two adjacent CL points in the TCS are marked as $M^{T C S}\left(u_{a, b}, v_{a, b}, w_{a, b}\right)$ and $M^{T C S}\left(u_{a, b+1}, v_{a, b+1}, w_{a, b+1}\right)$, respectively. Through the Eq. (5) in Section 2.1, there must be two corresponding adjacent points $M^{W C S}\left(x_{a, b}, y_{a, b}, z_{a, b}\right)$ and $M^{W C S}\left(x_{a, b+1}, y_{a, b+1}, z_{a, b+1}\right)$ in the WCS. Thus, the instantaneous feed vector (velocity vector) of point $\mathrm{M}$ could be described as follows:

$$
F_{a, b}=\left[\left(x_{a, b+1}-x_{a, b}\right),\left(y_{a, b+1}-y_{a, b}\right),\left(z_{a, b+1}-z_{a, b}\right)\right]
$$

Once obtaining the normal and feed direction of the arbitrary point on the cutter surface, the cutter could be divided into three areas: the forward area, backward area, and envelope boundary, as the FCS area of a cutter is shown in Figure 4. The different areas are defined as the following Eq. (9).

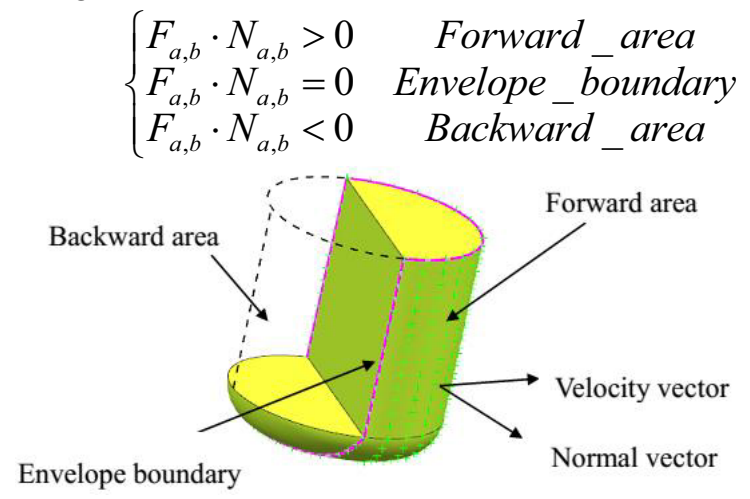

Figure 4. Feasible contact surface area of a cutter

The cutter may contact the in-process workpiece through the forward area and envelope 
boundaries. The combination of forward area and envelope boundaries determine the feasible contact surface of cutter. Finally the CWE areas are generated when the FCS engages the IPW during the

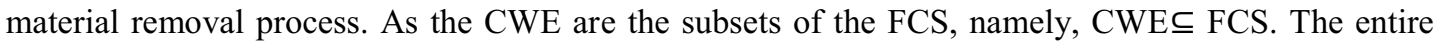
CWE extraction process is described clearly.

\section{Simulation results}

\subsection{Loop module for 5 -axis flank milling}

The cutter swept volume is generated by the cutter movement along the toolpath that can be obtained from the CAM module. As the loop diagram of the CWE model shown in Figure 5, when the cutter swept volume is calculated, the material removal volume can be simulated through a boolean subtraction with the raw stock. Then the CWE area can be extracted by the proposed method in Section 2.

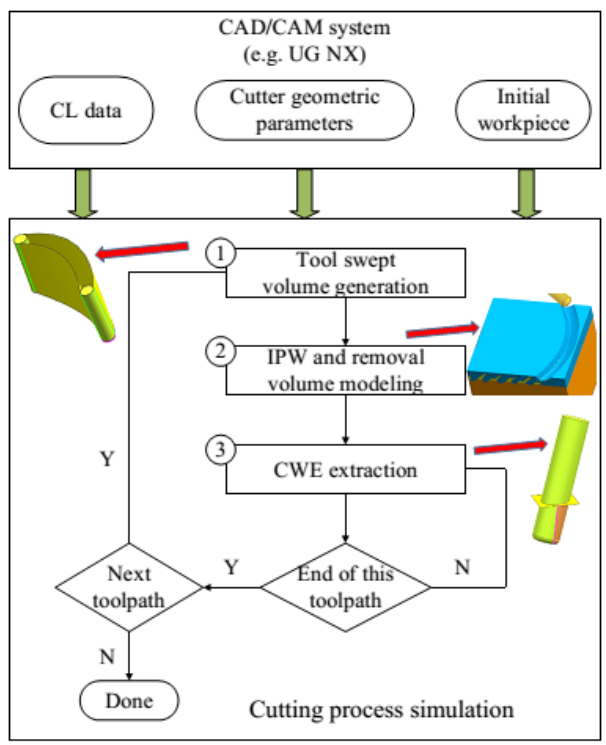

Figure 5. Loop of the CWE model

To compute the CWE area at a given CL point, the cutter body is intersected with some slicing planes which results in arcs or rings at each height increment in the TCS. These arcs/rings are then intersected with the IPW. The intersection results in several engagement curves. The starting and the end coordinates of these curves define the engagement points. Ultimately, engagement points are used to calculate the entrance and exit angles with respect to the plane of feed and cross-feed direction in the TCS. When these steps are done, the entire process should be updated including the IPW, cutter, and CL points. In each cycle firstly the cutter body at the current CL(i) point and the swept volume is subtracted from the IPW. Next step, the CL point counter for the loop is incremented by one and the cutter moves to the $\mathrm{CL}(\mathrm{i}+1)$ point. As a result, the IPW is updated and becomes ready for the CWE computation of the remaining CL points. Finally, the cutter body is created to be used again in the next iteration.

\subsection{Compute the engagement angle}

The CWE area and engagement angle computation form the main part of the proposed model. It also consumes most of the computational cost. The cutter body is sliced into a certain number of discs as is shown in cyan-yellow planes in Figure 6, in which they are shown as the removal volume after 
boolean operation between the cutter and workpiece. During this process, desired number of planes are created perpendicular to the tool axis at each plane height. To create these planes, normal direction and reference direction, which are the same with the cutter, are required.

These planes are perpendicular to the normal direction of the cutter at a given CL point and start from the tool tip point extending until the specified height of the cutter with very small height increments. These planes will be applied in combination with the cutter body for creating the cutter discs. Then, the slicing planes and the cutter body are intersected by the "intersect surface" function. Each intersection operation will result in a disc/arc lying on the corresponding plane, as the CWE calculation is shown in Figure 6.

The discs/arcs are later intersected with the copy of IPW. The IPW is duplicated to preserve the original IPW to be used in the following iterations [9]. The intersection results in an arc for single engagement or multiple arcs for multiple engagement that are in Figure 6 and Figure 7 . So the solid analytical model is capable of detecting the frequently occurring multiple engagement conditions in 5axis flank milling.

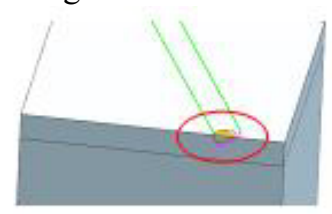

(a) The $1^{\text {st }} \mathrm{CL}$ point in milling process

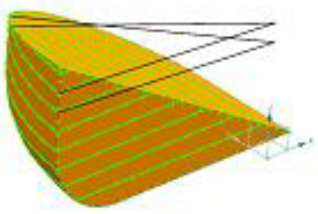

(d) Calculation of the CWE and engagement angle

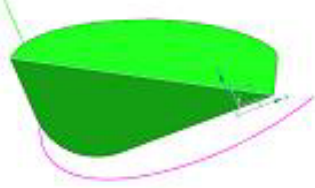

(b) The removal volume by boolean operation

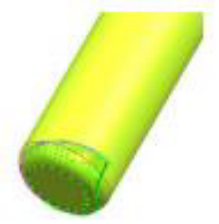

(e) The CWE of the $1^{\text {at }}$ cutting, $11^{\text {t }} \mathrm{CL}$ point

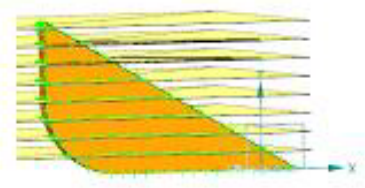

(c) Slicing planes, intersection points and curves of the IPW

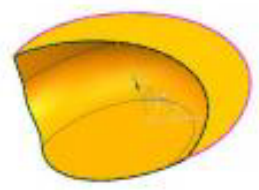

(f) The removal volume of the $11^{\text {th }} \mathrm{CL}$ point

Figure 6. Calculation of the CWE and engagement angle

The coordinate values of the start and end points from each arc are extracted and stored. They are called engagement points and are used in angle calculation. The angle between vector pointing to the current engagement point from the cutting center and the cross-feed direction vector is calculated. The entrance and exit angles are computed accurately by utilizing the inverse transformation of Eq. (5) to view them in the tool axis direction. This allows for the engagement angles to be measured with respect to the cross-feed direction. The calculated entrance and exit angles for each disc/arc are saved as CWE map to be used as a boundary condition for the cutting force prediction, as the CWE engagement angles are shown in Figure 7 and Table 1.

Table 1. CWE output of the $1^{\text {st }} \mathrm{CL}$ point (entrance (En) and exit (Ex) angles)

\begin{tabular}{cccc}
\hline CL point & Arc No. & En angle/degree & Ex angle/degree \\
\hline 01 & 1 & 8.997 & 107.393 \\
01 & 2 & 6.952 & 93.554 \\
01 & 3 & 6.534 & 84.637 \\
01 & 4 & 6.364 & 76.345 \\
01 & 5 & 6.335 & 67.799 \\
01 & 6 & 6.367 & 58.524 \\
01 & 7 & 6.399 & 48.208 \\
01 & 8 & 6.431 & 35.834 \\
01 & 9 & 6.463 & 17.253 \\
\hline
\end{tabular}

Additionally, in order to assess the performance of the proposed method, the output engagement angles of CWE model, i.e. Table 1, could be acted as an input in a cutting force model in research $[1,8,12]$. The engagement angles are utilized as the upper and lower boundaries $\left(\phi_{e n}, \phi_{e x}\right)$ in Eq. $(10)$ 
to estimate the cutting force. Here $d F_{x, y, z}(\phi)$ is the elemental cutting force in $\mathrm{X}, \mathrm{Y}$ and $\mathrm{Z}$ directions as a function of angular position of a cutter. Validation can be proved by comparing the simulated force data with the experimental force data that can be measured by a dynamometer. The simulation and experiment should be under the same condition of process parameters.

$$
F_{x, y, z}=\int_{\phi_{e n}}^{\phi_{e x}} d F_{x, y, z}(\phi) d \phi
$$

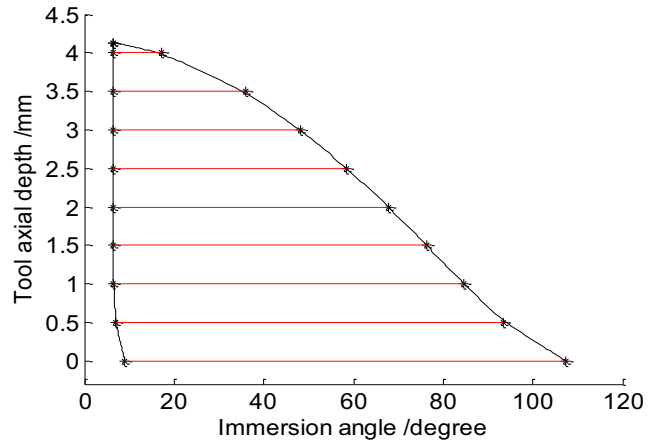

(a)

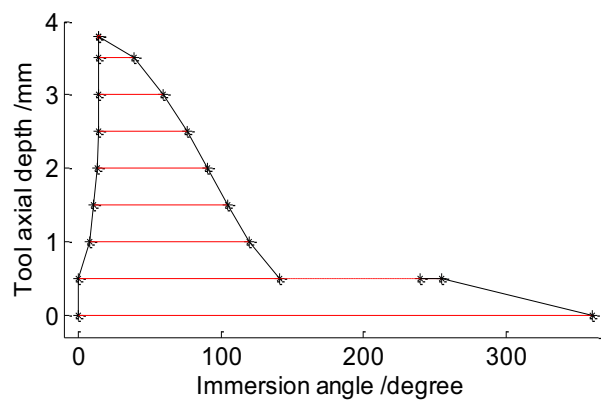

(b)

Figure 7. The CWE and engagement angle of the $1^{\text {st }}$ and $11^{\text {th }} C L$ points

\section{Conclusion}

The aim of this paper is introducing a solid analytical model to extract the CWE in 5-axis flank milling. The CL data and cutter geometry parameters are firstly generated from CAM, then the boolean subtraction operations are performed to obtain the possible CWE between the cutter swept volume and the raw workpiece, and the CWE and engagement angles are extracted finally. The proposed model can be integrated into commercial CAM applied to feedrate scheduling, tool orientation optimization and prediction of cutting force in 5-axis milling.

\section{Acknowledgments}

The research is supported by the National key R\&D project-the Construction, Reference Implementation and Verification Platform of Reconfigurable Intelligent Production System, China (Grant No. 2017YFE0101400).

\section{References}

1. $\quad$ Ferry W. B, Altintas Y. J MANUF SCI E-T ASME, 130 (1), 339-353 (2008).

2. Kim G. M, Cho P. J, Chu C. N. INT J MACH TOOL MANU, 40 (2), 277-291 (2000).

3. Lazoglu I, Boz Y, Erdim H. CIRP ANN-MANUF TECHN, 60 (1), 117-120 (2011).

4. Erdim H, Sullivan A. Procedia Cirp, 8, 438-443 (2013).

5. Altintas Y, Kersting P, Biermann D, et al. CIRP ANN-MANUF TECHN, 63 (2), 585-605 (2014).

6. Aras E, Albedah A. INT J ADV MANUF TECH, 73 (9-12), 1351-1362 (2014).

7. Chiou C. J, Lee Y. S. INT J MACH TOOL MANU, 42 (14), 1497-1507 (2002).

8. Zhang X, Yu T, Wang W. INT J ADV MANUF TECH, 74 (9-12), 1611-1624 (2014).

9. Yigit I. E, Lazoglu I, S. E. L. K. Procedia Cirp, 31, 179-184 (2015).

10. Ju G, Song Q, Liu Z, et al. INT J ADV MANUF TECH, 80 (5-8), 1297-1310 (2015).

11. Si H, Wang L, Zhang J, et al. INT J ADV MANUF TECH (9), 1-13 (2017).

12. Boz Y, Khavidaki S. E. L, Erdim H, et al. in Machining of Complex Sculptured Surfaces. Springer, 67-125 (2012). 